\title{
Characterization of putative proteins encoded by variable ORFs in white spot syndrome virus genome
}

\author{
Cayro de Macêdo Mendes ${ }^{1,2}$, Diego Gomes Teixeira ${ }^{3}$, João Paulo Matos Santos Lima ${ }^{2,3}$ and \\ Daniel Carlos Ferreira Lanza ${ }^{1,2,3^{*}}$
}

\begin{abstract}
Background: White Spot Syndrome Virus (WSSV) is an enveloped double-stranded DNA virus which causes mortality of several species of shrimp, being considered one of the main pathogens that affects global shrimp farming. This virus presents a complex genome of $\sim 300 \mathrm{~kb}$ and viral isolates that present genomes with great identity. Despite this conservation, some variable regions in the WSSV genome occur in coding regions, and these putative proteins may have some relationship with viral adaptation and virulence mechanisms. Until now, the functions of these proteins were little studied. In this work, sequences and putative proteins encoded by WSSV variable regions were characterized in silico.
\end{abstract}

Results: The in silico approach enabled determining the variability of some sequences, as well as the identification of some domains resembling the Formin homology 2, RNA recognition motif, Xeroderma pigmentosum group D repair helicase, Hemagglutinin and Ankyrin motif. The information obtained from the sequences and the analysis of secondary and tertiary structure models allow to infer that some of these proteins possibly have functions related to protein modulation/degradation, intracellular transport, recombination and endosome fusion events.

Conclusions: The bioinformatics approaches were efficient in generating three-dimensional models and to identify domains, thereby enabling to propose possible functions for the putative polypeptides produced by the ORFs wsv129, wsv178, wsv249, wsv463a, wsv477, wsv479, wsv492, and wsv497.

Keywords: ORF75, ORF94, ORF125, Uncharacterized proteins, VNTR regions

\section{Background}

White Spot Syndrome Virus (WSSV) is an enveloped double-stranded DNA virus recognized for its great impact on global shrimp farming and for the complexity of its $\sim 300 \mathrm{~kb}$ genome [1]. To date, little is known about the function of most of the $\sim 184$ WSSV predicted proteins since they have no homology with known sequences in the repositories [2]. Although most of these proteins present high identity among different WSSV isolates, variations are present in WSSV genome coding regions, including two genomic deletions occurring between

\footnotetext{
*Correspondence: danielclanza@gmail.com

${ }^{1}$ Applied Molecular Biology Lab - LAPLIC, Department of Biochemistry,

Federal University of Rio Grande do Norte, Natal, RN, Brazil

${ }^{2}$ Postgraduate Program in Bioinformatics, Federal University of Rio Grande do

Norte, Natal, RN, Brazil

Full list of author information is available at the end of the article
}

ORFs wsv461/wsv464 (14/15) and ORFs wsv77/wsv502 (23/24), and a variable number of tandem repeats (VNTRs) occurring within wsv129 (ORF75), wsv178 (ORF94) and wsv249 (ORF125) [3]. These variable regions have been used as molecular markers to identify viral variants [4-7]. Some studies have already indicated that these variable regions may have some relationship with the viral evolution and infection phenotype $[8,9]$, however there are still no direct correlations between the function of these putative products and virulence, mainly due to the lack of information about the functions of these proteins.

Computational tools have proven to be efficient for functionally characterizing proteins at low cost in a shorter time, thus enabling the analysis of some targets which cannot be evaluated in vitro, such as membrane proteins $[10,11]$ or proteins involved in viral infection 
mechanisms [12-15]. In this work, the putative proteins encoded by variable regions in the WSSV genome were structurally and functionally characterized using bioinformatics tools, and possible functions for these proteins were inferred.

\section{Methods}

Available nucleotide sequences corresponding to the variable regions from WSSV different isolates were retrieved from GenBank and subjected to a multiple alignment through MAFFT [16], and some adjustments were made by manual editing. Repeat units of each isolate were annotated using Geneious version 11.0.3 [17] and aligned against a reference sequence for annotation of polymorphism sites. Polymorphism sites were visualized by WebLogo [18].

Remote homologies between protein sequences were identified using BLAST tools (BLASTp, PSIBLAST and PHIBLAST) against non-redundant databases [19, 20]. Searches based on Hidden Markov Model profiles were also performed through JACKHMMER, hmmscan, HHBlits and HHPred [21-23].

Protein sequences were submitted in an iterative search to generate tertiary structure models using HHBlits (2-4 iterations) against the Uniclust30 database [24]. The best hits were selected from the generated output and submitted on HHPred against the PDB70 database. HHPred's best hits were used as templates to generate the structural models. The tertiary structure was modeled with PHYRE2, ITASSER, Swiss-Model and Modeller [25-28]. All models were based on the sequences of China 01 isolate (KT995472.1).

Secondary structures and threading predictions were also generated using PSIPRED and pGenThreader, respectively $[29,30]$. The obtained models were evaluated by Molprobity [31] evaluating the parameters clashscore, hydrogen bonds, van der walls contacts, geometry, rotamers, $C \beta$ deviations and cis-peptides. Ramachandran plots were generated by pyRAMA 2.0 [32]. All images from the models were generated by Chimera [33]. The validation of the structural models was performed through Verify3D which evaluates the compatibility of a three-dimensional model based on the 3D-1D scores that consists of the statistical preference of each of the amino acid residues that make up the model.

Structures used as templates for protein modeling were obtained from the Protein Data Bank (PDB): U1 small nuclear ribonucleoprotein (U1 snRNP, PDB: 4PKD), XPD repair helicase of Thermoplasma acidophilum (PDB: 4A15), Influenza hemagglutinin HA2 subunit (PDB: 1QU1), Ankyrin Repeat (PDB: 4HDB), RNF4 RING (PDB: 4AP4), Formin mDial Structure (PDB: 3OBV). Disordered regions, coiled coils, transmembrane regions and signal peptides were predicted using Foldindex, Coils, TMHMM and SignalP [34-36], respectively. SMART, CDD, ScanProsite and Eukaryotic Linear Motif (ELM) were used to detect conserved domains, patterns and motifs [37-40].

\section{Results}

Only characterization results with highest confidence levels based on the evaluation of the protein models are presented in this section. The pipelines that presented the best results for each model generated in this study are summarized in Table 1. The validation of the models was also performed using Verify3D. These results are presented in the Additional file 1.

\section{Characterization of some ORFs which occur in wsv461/ wsv464 and wsv477/wsv502 clusters}

The alignment of sequences corresponding to wsv461/ wsv464 and wsv477/wsv502 clusters revealed insertions of approximately $5 \mathrm{~Kb}$ and $13 \mathrm{~Kb}$, respectively. The wsv $461 /$ wsv464 cluster contains up to 6 ORFs (wsv461, wsv463a, wsv463b, wsv463c, wsv463d, wsv464), as detailed in Fig. 1a and Table 2. This cluster is truncated in most isolates, lacking these 6 ORFs. The insertions of the WSSV-CN02 and WSSV-TW isolates have wsv461 and wsv463 linked as a single coding region.

The number of putative coding regions in wsv477/ wsv502 cluster is higher comprising 13 ORFs, (wsv477, wsv479, wsv482, wsv484, wsv486, wsv489, wsv490, wsv492, wsv493, wsv495, wsv497, wsv500, wsv502), as detailed in

Table 1 Pipelines used to generate each of the models presented in this study. The numbers represent the sequence in which each software was used

\begin{tabular}{|c|c|c|c|c|c|c|c|}
\hline Model & HHBLITS & HHPRED & Modeller & Swiss-model & Phyre2 & ITASSER & PSIPRED \\
\hline wsv249 (ANK) & 1 & 2 & - & 3 & - & - & - \\
\hline wsv249 (RING) & 1 & 2 & - & 3 & - & - & - \\
\hline wsv463a & 1 & 2 & 3 & - & - & - & - \\
\hline wsv477 & 1 & 2 & 3 & - & - & - & - \\
\hline wsv479 & - & - & - & 2 & - & - & 1 \\
\hline wsv492 & - & 1 & - & 2 & - & - & - \\
\hline Wsv497 & - & - & - & 2 & - & - & 1 \\
\hline
\end{tabular}




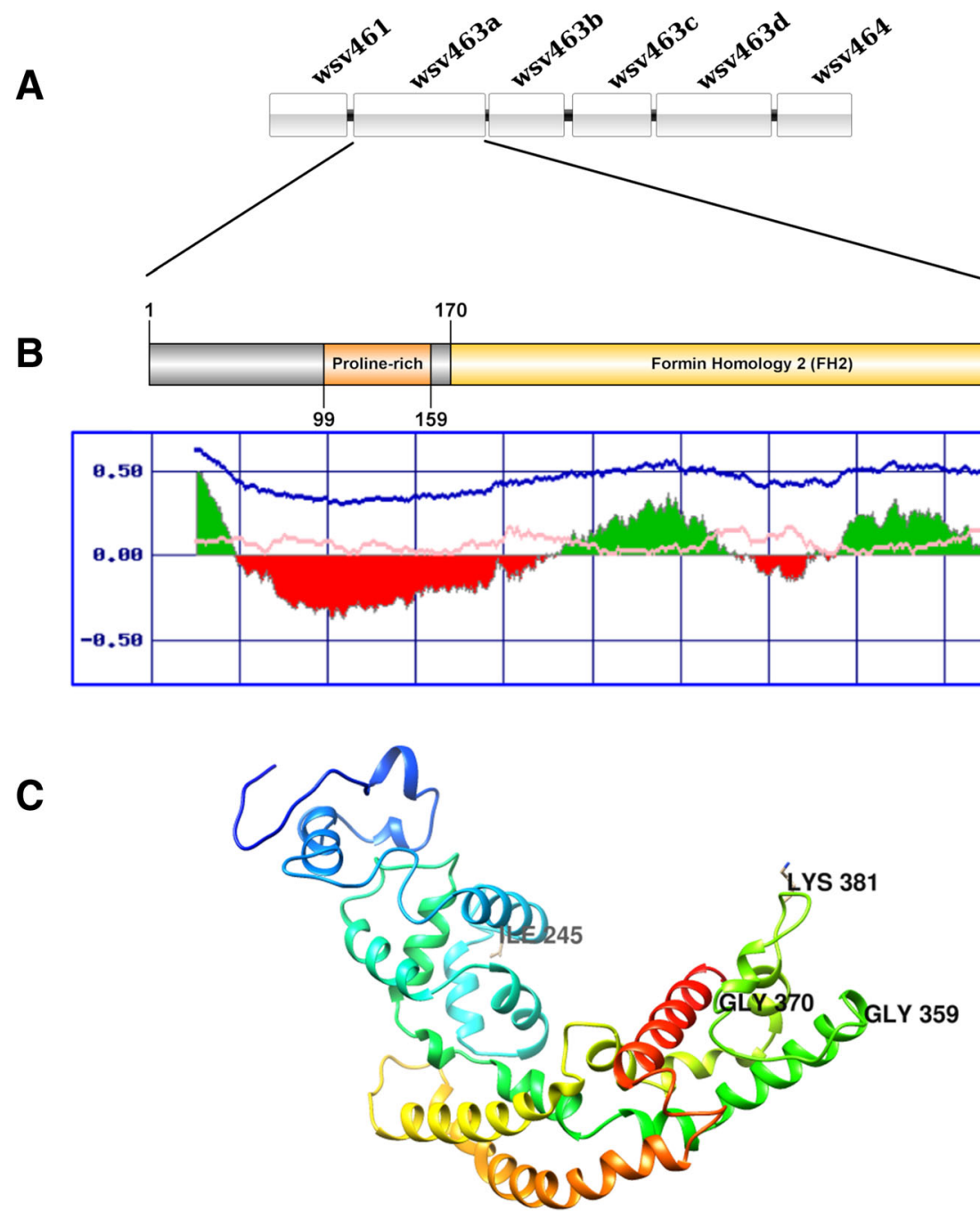

Fig. 1 Structural analysis of the putative protein encoded by ORF wsv463a (ORF14/15). a Schematic representation of wsv461/wsv464 cluster. $\mathbf{b}$ Schematic representation of the putative protein encoded by wsv463a, FH1 domain corresponding to the proline rich region (orange) and Formin homology 2 domain are shown in yellow. The graph below the scheme indicates the folded (green) and unfolded (red) regions predicted by Foldlndex, the $x$-axis indicates the amino acid positions and the $y$-axis represents the probability scores. c Three-dimensional model of the wsv463a sequence corresponding to Formin Homology 2 domain. Conserved subdomains (Lasso, Linker, Knob, Coiled Coil and Post) and amino acids of the typical FH2 domain are indicated

Fig. 2a and Table 3. The characterization results of each coding region for both clusters is presented below.

\section{ORF wsv463a}

The ORF wsv463a, a putative coding sequence located in wsv461/wsv464 cluster (Fig. 1a), presented a proline rich domain located in an unfolded portion of the predicted protein (positions 99-159) and a larger domain similar to the Formin Homology 2 (FH2) between positions 170-508 (Fig. 1b). The 3D model of the larger structured domain confirms the alpha-helical structure of $\mathrm{FH} 2$, composed by five alpha-helical subdomains (Lasso, Linker, Knob, Coiled Coil and Post) (Fig. 1c). The validation tests, main scores and the ramachandran plot corresponding to the 3D model are presented in Additional file 2.

\section{ORF wsv477}

The ORF wsv477 is the first coding region in wsv477/ wsv502 cluster (Fig. 2a). The putative product of wsv477 presents a domain homologous to a RNA Recognition Motif (RRM) in positions 70-139 (Fig. 2b), and a "Zinc-Finger" domain in its C-terminal region. The 3D model of the RRM domain revealed a tertiary structure with two alpha-helices and three beta-sheets following a $\beta 1 \alpha 2 \beta 2 \beta 3 \alpha 2$ pattern, which corresponds to the typical RRM $\beta 1 \alpha 2 \beta 2 \beta 3 \alpha 2 \beta 4$ structure (Fig. $2 c$ and Additional file 3). Aromatic residues involved in RNA 
Table 2 Characterization of sequences corresponding to ORF wsv461/wsv464 (ORF14/15) cluster obtained from different WSSV isolates

\begin{tabular}{|c|c|c|c|c|}
\hline $\begin{array}{l}\text { Accession } \\
\text { Number }\end{array}$ & $\begin{array}{l}\text { Description } \\
\text { (Country) }\end{array}$ & $\begin{array}{l}\text { Insertion lentgh } \\
\text { (bp) }\end{array}$ & $\begin{array}{l}\text { Number of putative ORFs } \\
\text { (including wsv461 and wsv464) }\end{array}$ & Putative ORFs \\
\hline AF440570.1 & Taiwan & 506 & 2 & (wsv461 f, wsv464) \\
\hline AF332093.3 & China & 257 & 3 & (wsv461* ${ }^{*}$ wsv463 f,wsv464) \\
\hline AF369029.2 & Thailand & - & 2 & (wsv461, wsv464*) \\
\hline JX515788.1 & South Korea & - & 1 & (Wsv464) \\
\hline KR083866.1 & Egypt & 257 & 3 & (wsv461* ,wsv463 f,wsv464) \\
\hline KT995472.1 & China 01 & 4999 & 6 & $\begin{array}{l}\text { (wsv461, wsv463a, wsv463b, wsv463c, } \\
\text { wsv463d,wsv464) }\end{array}$ \\
\hline KT995470.1 & China 02 & 506 & 2 & (wsv461 f, wsv464) \\
\hline KT995471.1 & China 03 & - & 2 & (wsv461*, wsv464*) \\
\hline KY827813.1 & China 04 & - & 2 & $($ wsv461*, wsv464*) \\
\hline MF784752.1 & Brazil & - & 2 & (Wsv461, Wsv464*) \\
\hline KR083844.1 & Egypt 09 & 4894 & 6 & $\begin{array}{l}\text { (wsv461, wsv463a, wsv463b, } \\
\text { wsv463c, wsv463d,wsv464) }\end{array}$ \\
\hline KF771904.1 & USA Texas & 4894 & 6 & $\begin{array}{l}\text { (wsv461, wsv463a, wsv463b, } \\
\text { wsv463c, wsv463d,wsv464) }\end{array}$ \\
\hline AY753327.1 & Thailand 96 ॥ & 5025 & 7 & $\begin{array}{l}\text { (wsv461, wsv463a t, wsv463b, } \\
\text { wsv463c, wsv463d,wsv464) }\end{array}$ \\
\hline KR083865.1 & Egypt 14 & 137 & 2 & $\left(\right.$ wsv461 ${ }^{*}$, Wsv464*) \\
\hline KF771899.1 & USA DC97 & 137 & 2 & $\left(\right.$ wsv461 ${ }^{*}$, wsv464*) \\
\hline KR083846.1 & EGYPT 11 & 1136 & 2 & $\left(\right.$ wsv461* ${ }^{*}$ wsv464*) \\
\hline KF771902.1 & USA Texas 2 & 1136 & 2 & $\left(\right.$ wsv461 ${ }^{*}$, wsv464*) \\
\hline KX501221.1 & Thailand 12 & - & 2 & (wsv461*, wsv464*) \\
\hline EU327501.1 & India 05 & - & 2 & $\left(\right.$ wsv461 ${ }^{*}$, wsv464*) \\
\hline MG702567.1 & INDIA AP4RU & - & 2 & (wsv461*, Wsv464*) \\
\hline HQ257380.1 & Mexico Mx-F & 335 & 2 & $\left(\right.$ wsv461 ${ }^{*}$, wsv464*) \\
\hline HQ257382.1 & Mexico Mx-C & 335 & 2 & (wsv461*, Wsv464*) \\
\hline HQ257383.1 & Mexico Mx-G & 335 & 2 & $\left(\right.$ wsv461* ${ }^{*}$ wsv464*) \\
\hline MF768985.1 & Australia & 171 & 2 & $\left(\right.$ wsv461 ${ }^{*}$, Wsv464*) \\
\hline KX501220.1 & Thailand TH-14 & - & 2 & $\left(\right.$ wsv461* ${ }^{*}$ wsv464*) \\
\hline KU746817.1 & China FCG2-14 & - & 2 & (wsv461*, wsv464*) \\
\hline EF468499.1 & India IN-07 & 335 & 2 & $\left(\right.$ wsv461* ${ }^{*}$ wsv464*) \\
\hline
\end{tabular}

*Partial sequences

f Fusion of two sequences

t Two ORFS formed in wsv463a (occurs exclusively on WSSV TH-96-II)

binding remain conserved in the central strands of the proposed protein, which are composed of Phe74 at position 2 of the $\beta 1$, Tyr118 at position 5 of $\beta 3$ and Phe116 position 3 of $\beta 3$ (Fig. 2c).

\section{ORFs wsv479 and wsv497}

The ORFs wsv479 and wsv497 are also located in the wsv477/wsv502 cluster (Fig. 2a). These ORFs produce putative proteins that are similar in amino acid composition, size and folding, suggesting that they have similar functions (Fig. 2d, e, f and g). It was not possible to infer any function by sequence homology. By using threading approaches it was possible to determine that the tertiary structure of these two ORFs present similar folding to Xeroderma pigmentosum group D repair helicase (XPD) (Fig. 2e, g and Additional files 4 and 5). XPD belongs to the helicase superfamily 2 and a component of transcription factor IIH (TFIH), which is associated with Nuclear Excision Repair pathway (NER), catalyzing the opening of the double helix around the damaged site, providing access to NER factors in a ATP-dependent process [41]. The XPD helicase consists of two motor domains called HD1 and HD2, an arch domain and an iron-sulfur 


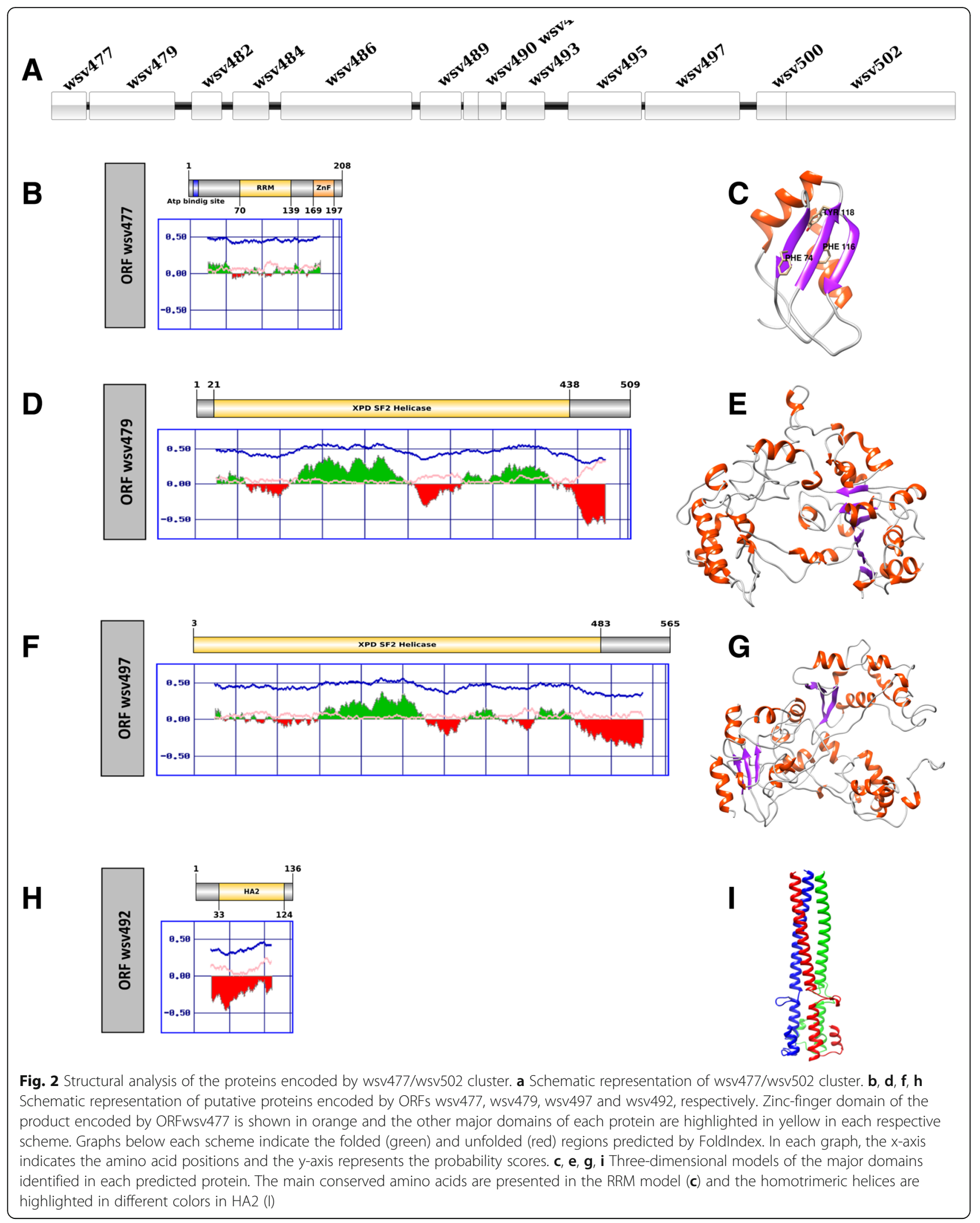


Table 3 Characterization of sequences corresponding to ORF wsv477/Wsv502 (ORF23/24) cluster, obtained from different WSSV isolates

\begin{tabular}{|c|c|c|c|c|}
\hline $\begin{array}{l}\text { Accession } \\
\text { Number }\end{array}$ & $\begin{array}{l}\text { Description| } \\
\text { (Country) }\end{array}$ & $\begin{array}{l}\text { Insertion } \\
\text { lentgh (bp) }\end{array}$ & $\begin{array}{l}\text { Number of putative ORFs (including } \\
\text { wsv477 and wsv502) }\end{array}$ & Putative ORFs \\
\hline AF440570.1 & Taiwan & 12,613 & 13 & $\begin{array}{l}\text { (wsv477, wsv479, wsv482, wsv484, wsv486, wsv489, } \\
\text { Wsv490, wsv492, wsv493, wsv495, wsv497, wsv500, wsv502) }\end{array}$ \\
\hline AF332093.3 & China & 11,451 & 13 & $\begin{array}{l}\text { (Wsv477, Wsv479, Wsv482, Wsv484, Wsv486, Wsv489, } \\
\text { Wsv490, Wsv492, Wsv493, WSV495a, WSV497, Wsv500, Wsv502) }\end{array}$ \\
\hline AF369029.2 & Thailand & 81 & 2 & (wsv477, wsv502) \\
\hline JX515788.1 & South Korea & 6959 & 8 & (wsv477, wsv479, wsv482, wsv484, wsv486, wsv500, wsv502) \\
\hline KR083866.1 & Egypt & 11,451 & 13 & $\begin{array}{l}\text { (wsv477, wsv479, wsv482, wsv484, Wsv486, Wsv489, } \\
\text { Wsv490, Wsv492, Wsv493, Wsv495a, Wsv497, Wsv500, Wsv502) }\end{array}$ \\
\hline KT995472.1 & China 01 & 12,536 & 13 & 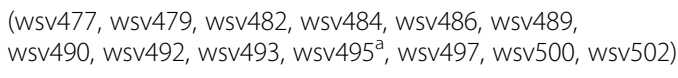 \\
\hline KT995470.1 & China 02 & 6718 & 8 & (wsv477, wsv479, wsv482, wsv484, wsv486, wsv500, wsv502) \\
\hline KT995471.1 & China 03 & 1643 & 4 & (wsv477, wsv479a , wsv500, wsv502) \\
\hline KU216744.1 & Mexico & 98 & 2 & (wsv477, wsv502) \\
\hline KY827813.1 & China 04 & 1643 & 4 & (wsv477, wsv479a , wsv500, wsv502) \\
\hline MG264599.1 & Brazil & 12,090 & 13 & $\begin{array}{l}\text { (wsv477, wsv479, wsv482, wsv484, wsv486, Wsv489, } \\
\text { wsv490, wsv492, wsv493, Wsv495, Wsv497, Wsv500, wsv502) }\end{array}$ \\
\hline
\end{tabular}

${ }^{\text {aPartial sequences }}$

cluster (FeS) superimposed on HD1. The process of binding XPD to DNA is through the HDR2 domain [42].

\section{ORF wsv492}

The wsv492 putative product demonstrated high similarity with the HA2 subunit of the hemagglutinin influenza virus (Fig. 2h, i). The 3D model shows a HA2-like subunit formed by a triple-helical chain (Fig. 2i and Additional file 6).

\section{Characterization of ORFs comprising VNTRs}

The alignment of sequence sets for ORFs wsv129, wsv178 and wsv249 revealed that high and less variable regions are wsv129 and wsv249 respectively, considering the VNTR size and the total number of analyzed sequences (Table 4). It has been observed that repeat units (RUs) contain few polymorphic sites in all cases. Substitutions in wsv178 were observed occurring in positions 1,36 and 48 . Wsv249 has substitutions in positions 2 , $9,12,27,50,53$ and 61 , with the last three occurring at a higher frequency. Wsv129 has two types of repeat units, the most frequent having $45 \mathrm{bp}$ and a $57 \mathrm{bp}$ repeat intercalating the $45 \mathrm{bp}$ RUs. Indels occur more frequently in wsv178 and wsv249.

\section{ORF wsv129}

Secondary structure predictions revealed small transmembrane helices in the $\mathrm{N}$-terminal region (positions 50-72) which coincide with a structured region of the wsv129 predicted protein (Fig. 3a). Curiously, several coiled coils coded by the $57 \mathrm{bp}$ repeat units and nuclear localization signals (NLS) are predicted at the end of each repeat in VNTR region (Fig. 3a).

\section{ORF wsv178}

Small transmembrane helices were also predicted in the $\mathrm{N}$-terminal region of wsv178 putative product (positions 7-29) coinciding with the unique folded region of the protein (Fig. 3b). Curiously, a cleavage site was predicted between residues 26 and 27 which could separate the product encoded by the VNTR region from the transmembrane portion. The presence of putative nuclear localization signals was also observed at the beginning of each repeat unit.

Table 4 Repeat units in WSSV VNTRS

\begin{tabular}{|c|c|c|c|c|c|c|}
\hline VNTRs & $\begin{array}{l}\text { RU length } \\
\text { (bp) }\end{array}$ & $\begin{array}{l}\text { Number of aligned } \\
\text { sequences }\end{array}$ & $\begin{array}{l}\text { Number of different } \\
\text { sizes }\end{array}$ & $\begin{array}{l}\text { Minimum number } \\
\text { of RUs }\end{array}$ & $\begin{array}{l}\text { Maximum number } \\
\text { of RUs }\end{array}$ & $\begin{array}{l}\text { Most frequent } \\
\mathrm{RU}\end{array}$ \\
\hline wsv129 (ORF75.a) & 45 & 39 & 13 & 2 & 21 & 11 \\
\hline wsv 129 (ORF75.b) & 57 & 39 & 5 & 1 & 5 & 3,4 \\
\hline wsv178 (ORF94) & 54 & 43 & 12 & 1 & 18 & 6 \\
\hline wsv249 (ORF125) & 69 & 34 & 5 & 2 & 7 & 4 \\
\hline
\end{tabular}




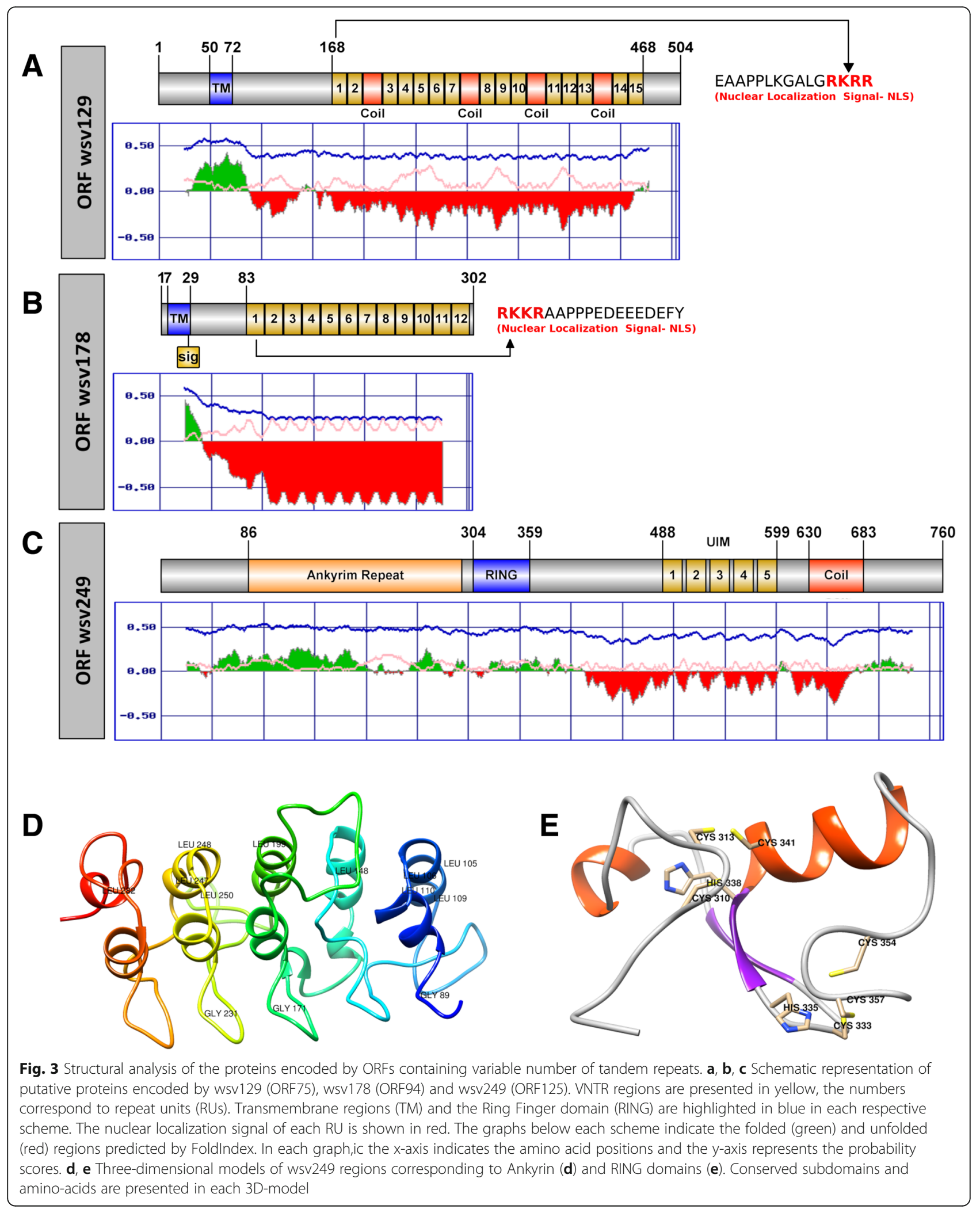




\section{ORF wsv249}

The product generated by wsv 249 has a more structured chain compared to the other ORFs that contain VNTR regions (Fig. 3c). The characterization by remote homology and fold recognition revealed that the first 300 residues of the N-terminal region correspond to a Ankyrin repeat (ANK) motif (Fig. 3d and Additional file 7). It was also possible to identify a RING-H2 domain immediately after the ANK domain region. The 3D model of the RING-H2 domain is shown in Fig. 3e, as well as the main conserved amino acids. The validation tests of this three-dimensional model are presented in Additional file 8.

Some glycine residues $(89,171,231)$ located in the second beta sheet as well as leucine residues located in second alpha-helix $(105,106,109,110,148,199,247$, $248,250,282$ ) of ANK motif remain in conserved positions in the proposed model. The typical tetrapeptide TPLH was not observed in sequence due to divergence at the primary structure level.

SMART data revealed that the tandem repeat units in wsv249, despite the high E-value (due to the small sequence length), corresponding to ubiquitin-interacting motifs (UIM) which consist of 20 residues alpha-helix of a X-Ac-Ac-Ac-Ac- $\Phi$-X-X-Ala-X-X-X-Ser-X-X-Ac-X-X-X-X consensus, where " $\Phi$ " corresponds to a hydrophobic residue, "Ac" is acidic residues and " $\mathrm{X}$ " can be any amino acid residue [43, 44]. Additionally, a RING-H2 was detected immediately next to the Ankyrin domain located between positions 310-357 of the protein (Fig. 3c, e), which has the Cys-X2-Cys-X(9-39)-Cys-X(1-3)-His-X(2-3)-His-X2-Cys-X (4-48)-Cys-X2-Cys pattern, where " $\mathrm{X}$ " comprises any amino acid.

\section{Discussion}

\section{wsv461/wsv464 and wsv477/wsv502 clusters}

The number of sequences with the truncated insertion is smaller in wsv477/wsv502 cluster when compared to the wsv461/wsv464. This observation is in accordance with previous results which demonstrate that some proteins encoded by wsv $477 /$ wsv502 cluster possibly have functional domains $[45,46]$.

\section{ORF wsv 463}

The protein encoded by ORFwsv463 presents formin characteristics. Formins consists of a family of proteins that regulates the elongation of unbranched actin filaments which are important in many cellular processes, including formation of actin cables, cytokinetic ring, filopodia and stress fibers [47, 48]. These processes are mediated by the FH2 domain located in the C-terminal region, which forms a stable hydrophobic ring-like hemidimer and binds the ends of actin filaments protecting from capping proteins [49, 50]. Each hemidimer has conserved residues that are directly related to actin binding consisting of an Ile located in the subdomain knob and Lys located in the lasso/post interface. Mutations within these conserved sites may compromise actin nucleation activity [51]. The glycine residues at positions 359 and 370 act on dimerization, as well as Ile245 and Lys381 acting in actin-binding remain conserved in the wsv463a predicted protein (Fig. 1c).

Formins also have a FH1 domain composed of polyproline, similar to what was observed in the wsv463a protein scheme (Fig. 1b). The FH1 domain is directly related to the interaction with profilin proteins during the actin elongation. The actin monomer binding protein profilin stimulates the actin assembly through binding FH1 and FH2 domains, increasing elongation speed [51, 52].

As an essential component of cellular cytoskeleton, actin can be manipulated by viruses into the host cells in many stages of its life-cycle, including entry, motility, nuclear and assembly [53], modulating the activity of actin binding proteins. Actin filaments provide mechanical force for viral pathogens to navigate within the host cell, causing changes in cellular shape [54]. HIV is able to navigate between dendritic cells through filopodia produced by formins [54]. Formin FHOD1 together with the small GTPase Rac1 of Vaccinia virus is associated with actin tail formation, acting in an integrated way with the $\mathrm{N}$-WASP-ARP2/3 pathway, thus being essential for Vaccinia virus motility and dissemination [55]. The fact that WSSV encodes formins may be related to regulation of host fibrous proteins involved in viral packaging and/or intracellular transport.

\section{ORF wsv 477}

ORF wsv477 was previously characterized as a $624 \mathrm{bp}$ immediate early gene encoding putative protein of the 208 amino acid, with an ATP/GTP binding site between positions 7-14 and a Cys2/Cys2 type Zinc-finger domain between residues 169-197 [56]. In addition, a miR-7 injection in WSSV infected shrimp could reduce the wsv477 expression and decrease the number of WSSV genome copies at 12 to $96 \mathrm{~h}$ post infection $[57,58]$. The results presented herein corroborate that the RRM domain in wsv 477 protein may be related to post transcriptional steps (splicing, pre-mRNA processing, RNA editing, translation regulation) which determine the efficiency of viral replication.

RRM can be found in all organisms, being found with greater abundance in proteins encoded by eukaryotes in multiple copies or in conjunction with other domains like "Zinc-Finger" domains of the $\mathrm{CCCH}$ or $\mathrm{CCHC}$ types, which can bind to RNA. This domain is involved in post-transcriptional events including splicing, pre-mRNA processing, RNA editing, translation regulation, and RNA degradation [59-63]. Some RRM proteins are involved in replicating RNA viruses, including 
heterogeneous nuclear ribonucleoprotein A1 (hnRNP A1) in Hepatitis $\mathrm{C}$ virus which interact with an RNAdependent RNA-polymerase and septin 6 , forming a replication complex [64]. The interface between RNA and RRM occurs through four conserved residues located in the central $\beta 1$ and $\beta 3$ (called RNP2 and RNP1, respectively) of the RRM, where nitrogenous bases of the RNA bind to the side chain of the localized aromatic amino acids in the $\beta 1$ (position 2 of RNP2) and in $\beta 3$ (position 5 of RNP1). The third aromatic residue at position $\beta 3$ (position 3 of RNP1) hydrophobically interacts with the two pentose rings of each nucleotide $[65,61]$.

\section{ORFs wsv479 and wsv497}

It has been previously described that wsv 479 and wsv497 sequences have a conserved VP9 domain (also known as ICP11) located in the N-terminal region [66] having a ferredoxin fold which has been suggested as a DNA recognition domain. Considering this and the presented results, these proteins probably have functions related to WSSV genome processing and recombination events.

\section{ORF wsv492}

The wsv492 putative protein probably has functions related to hemagglutinin. Hemagglutinins consist of glycoproteins which remain anchored in the viral envelope and mediates viral entry [67]. After protein synthesis, the sequence encoding hemagglutinin (HA0) undergoes a post-translational cleavage, producing the HA1 and HA2 subunits which form a homotrimeric structure. The HA1 subunit interacts with sialic acid, a monosaccharide present on the membrane surface mediating the endocytosis of the viral particle [68]. The HA2 subunit consists of a triple-helical hydrophobic structure associated with the $\mathrm{pH}$-induced fusion process, a mechanism by which the virus releases from the endosome and contacts the host cell cytosol.

\section{ORFs comprising VNTRs}

The RU profiles observed in VNTRs of ORFs wsv129, wsv178 and wsv249 coincide with those already previously described [69, 70]. Interestingly, the reading windows are maintained in even more variable VNTRs. It was not possible to obtain reliable three-dimensional models for the wsv129 and wsv178 putative products, since they have a large unstructured portion, as well as many charged amino acids.

\section{ORF wsv129}

The prediction of a large unstructured portion rich in coiled coils in association with the transmembrane domain in the wsv129 polypeptide indicates a structural function. In fact, 18 structural proteins located in the WSSV virion, including the protein encoded by wsv129, were previously detected by proteomic analysis [71]. In this same work, a temporal analysis showed that the wsv129 product is expressed late, at least $6 \mathrm{~h}$ after the WSSV infection.

NLS act by directing proteins into the cell nucleus and can be subdivided into two subclasses: monopartite, formed by a group consisting of a sequence $K(K / R) X$ $(K / R)$, or bipartite, which are formed by two groups of basic residues separated by a 10-12 amino acid linker which may vary [72]. The transport of macromolecules through the nucleus occurs through a nucleoporin protein complex called the nuclear pore complex (NPC). Proteins above $40 \mathrm{kDa}$ require a specific signal which will allow it to interact with carrier proteins that will facilitate its entry into the nucleus [73-75].

The binding of the carrier protein of the NLS is important for the release of the viral molecules into the nucleus. DNA viruses that infect animals replicate in the nucleus of the host cell. To drive the entry of the viral genome into the nucleus, large viruses normally release their DNA associated with structural proteins which are associated with nuclear localization signals [76].

Viral proteins containing NLS signals in tandem were not found in the literature. Since the mechanism of WSSV entry into the host cell nucleus is unknown, this may be an indication of a new type of entry mechanism which needs to be better investigated. On the other hand, it is not possible to rule out the possibility that these NLS signals are artifacts of the software analysis.

\section{ORF wsv178}

The structural data presented herein indicates that wsv129 and wsv178 are related to similar functions, as previously suggested [77]. These proteins may also have some adaptive function, considering that these two VNTR are the most variable, even among isolates from the same region.

\section{ORF wsv249}

Ankyrin repeat (ANK) is a motif composed of about 33 amino acid residues which are important in the modulation of several cell pathways mediating specific proteinprotein interactions; most of the protein sequences that exhibit these motifs usually consist of transcriptional regulators, modulators of cellular development and differentiation [78]. The ANK motif adopts a helix-loophelix structure in which two alpha-helices are arranged in an anti-parallel fashion and the loop protrudes out of the frame to facilitate the formation of hairpin-like beta-sheet with neighboring loops. The conserved tetrapeptide T-P-L-H (6-9) forms a closed curve starting at the first alpha-helix of the ANK [79]. Hydrogen bonds between the threonine hydroxyl groups and the histidine imidazolic ring contribute to ANK stability. The Val/Ile-Val-XXX 
(hypophilic)-Leu/Val-Leu-Leu motif (positions 17-22) located in second alpha-helix stabilize the overall framework of an ANK protein. Glycine residues are stored at position 4 located in the second beta sheet, and at 13 and 25 at the alpha-helix ending [80].

ANK is a motif found in abundance in eukaryotic species, but little is known about the presence of ANK motif in viral proteins, except for species of the poxvirus genus that present ANK motifs in the terminal regions of the proteins involved in the ubiquitination process [81, 82]. A PSI-BLAST of the ANK domain was performed against poxvirus sequences, and it was possible to observe hits for the ANK motif, despite the low coverage presented.

The ORF wsv249 product was previously characterized as a ubiquitin ligase, acting as a key element in the modulation of protein abundance within cells through the ubiquitin-dependent proteolysis mechanism [83, 84]. The activity of ubiquitin-ligase is governed by the RING-H2 domain. The presence of ANK in conjunction with RING-H2 reinforces the function of wsv249 in the modulation of WSSV host proteins.

\section{Conclusion}

The alignment of variable sequences revealed that the most and least variable regions are wsv129 and wsv 249 respectively, and that most of the already sequenced isolates did not present insertion in the wsv461/wsv464 cluster. The different approaches used were efficient in generating three-dimensional models and identifying domains, which enabled proposing functions for the putative polypeptides produced by the ORFs wsv249, wsv463a, wsv477, wsv479, wsv492, wsv497. The results indicate that these proteins are possibly involved in mechanisms related to protein modulation/degradation, intracellular transport, endosome recombination and fusion events. In addition, through the analysis of the secondary structure and characterization of the VNTR regions, it was possible to suggest that the products encoded by the ORFs wsv129 and wsv178 have structural function and may be involved in the WSSV adaptive mechanisms.

Considering that ORFs wsv463a, wsv479, wsv492 and wsv497 occur in a small number of WSSV isolates, their functions are not essential for the WSSV infection, or are being supplied by the cellular metabolism of the host. On the other hand, considering that wsv129, wsv178, wsv249 and wssv477 occur in all WSSV isolates and that sequence variations do not compromise the protein frame, their functions related to structural/packaging (wsv129, wsv178, wssv477) or in ubiquitination processes (wsv249) are possibly essential for viral replication and maintenance, and can be adaptive.

\section{Additional files}

Additional file 1: Verify3D evaluation of protein models (DOCX $16 \mathrm{~kb}$ ) Additional file 2: Quality scores of predicted model of Formin Homology 2 domain (FH2). (A) Global QMEAN scores generated by SwissModel; (B) Ramachandran plots generated by pyRAMA; (C) Molprobity score. (PDF 1607 kb)

Additional file 3: Quality scores of the RNA recognition motif predicted model. (A) Global QMEAN scores generated by Swiss-Model; (B) Ramachandran plots generated by pyRAMA; (C) Molprobity score. (PDF $1496 \mathrm{~kb}$ )

Additional file 4: Quality scores of the XPD Helicase (wsv479) predicted model. (A) Global QMEAN scores generated by Swiss-Model; (B) Ramachandran plots generated by pyRAMA; (C) Molprobity score. (PDF $1582 \mathrm{~kb}$ )

Additional file 5: Quality scores of the XPD Helicase (wsv497) predicted model. (A) Global QMEAN scores generated by Swiss-Model; (B) Ramachandran plots generated by pyRAMA; (C) Molprobity score. (PDF $1611 \mathrm{~kb}$ )

Additional file 6: Quality scores of the HA2 hemagglutinin predicted model. (A) Global QMEAN scores generated by Swiss-Model; (B) Ramachandran plots generated by pyRAMA; (C) Molprobity score. (PDF $1495 \mathrm{~kb}$ )

Additional file 7: Quality scores of the Ankyrin repeat domain (ANK) predicted model. (A) Global QMEAN scores generated by Swiss-Model; (B) Ramachandran plots generated by pyRAMA; (C) Molprobity score. (PDF $1557 \mathrm{~kb}$ )

Additional file 8: Quality scores of the RING-H2 domain predicted model. (A) Global QMEAN scores generated by Swiss-Model; (B) Ramachandran plots generated by pyRAMA; (C) Molprobity score (PDF $1469 \mathrm{~kb}$ )

\section{Abbreviations}

ANK: Ankyrim Repeat; CDD: Conserved Domains Database; ELM: Eukaryotic Linear Motif; FH1: Formin Homology 1; FH2: Formin Homology 2; FHOD1: FH1/FH2 domain-containing protein 1; HA: Hemagglutinin; HIV: Human Immunodeficiency Virus; hnRNP A1: Heterogeneous Nuclear Ribonucleoprotein A1; ICP: Infected Cell Protein; mDia1: Mammalian Diaphanous 1; miR-7: MicroRNA 7; NER: Nuclear Excision Repair; NLS: Nuclear Localization Signal; NPC: Nuclear Pore Complex; ORF: Open Reading Frame; PDB: Protein Data Bank; RNF4: RING Finger Protein 4; RNP: Ribonucleoprotein RRM: RNA Recognition Motif; RU: Repeat Units; TFIIH: Transcription Factor IH; U1 snRNP: U1 small nuclear ribonucleoprotein; UIM: Ubiquitin-Interacting Motif; VNTR: Variable Number Tandem Repeat; VP9: Viral Protein 9; WSSV: White Spot Syndrome Virus; XPD: Xeroderma Pigmentosum Group D

\section{Acknowledgments}

We would like to thank the Núcleo de Processamento de Alto Desempenho of the Universidade Federal do Rio Grande do Norte - NPAD / UFRN for access to the supercomputer.

\section{Funding}

This research received financial support from the Conselho Nacional de Desenvolvimento Científico e Tecnológico - CNPq (grant no: 409378/2016-0), and the Coordenação de Aperfeiçoamento de Pessoal de Nível Superior (CAPES).

\section{Availability of data and materials}

All data concerning this work are already available in the body of the text and figures, as well as in supplementary materials. It will not be necessary to make any data available through databases.

\section{Authors' contributions}

CMM performed the computational tests, elaborated the figures and assisted in writing the text. DGT assisted in the computational tests. JPMSL assisted in planning the research and executing the analyzes, and DCFL idealized the research, wrote the manuscript and helped in elaborating the figures. All authors read and approved the final manuscript. 


\section{Ethics approval and consent to participate}

Not applicable.

\section{Consent for publication}

Not applicable.

\section{Competing interests}

The authors declare that they have no conflict of interest.

\section{Publisher's Note}

Springer Nature remains neutral with regard to jurisdictional claims in published maps and institutional affiliations.

\section{Author details}

'Applied Molecular Biology Lab - LAPLIC, Department of Biochemistry, Federal University of Rio Grande do Norte, Natal, RN, Brazil. ${ }^{2}$ Postgraduate Program in Bioinformatics, Federal University of Rio Grande do Norte, Natal, RN, Brazil. ${ }^{3}$ Postgraduate Program in Biochemistry, Federal University of Rio Grande do Norte, Natal, RN, Brazil.

\section{Received: 10 October 2018 Accepted: 28 March 2019}

\section{Published online: 18 April 2019}

\section{References}

1. Verbruggen B, Bickley LK, van Aerle R, Bateman KS, Stentiford GD, Santos EM, et al. Molecular mechanisms of white spot syndrome virus infection and perspectives on treatments. Viruses. 2016;8:1-29.

2. van Hulten MCW, Witteveldt J, Peters S, Kloosterboer N, Tarchini R, Fiers M, et al. The white spot syndrome virus DNA genome sequence. Virology. 2001;286:7-22. https://doi.org/10.1006/viro.2001.1002.

3. Hoa TT, Hodgson RAJ, Oanh DT, Phoung NT, Preston NJ, Walker PJ. Genotypic variations in tandem repeat DNA segments between ribonucletide reductase subunit genes of White Spot Syndrome Virus (WSSV) isolates from Vietnam. Dis Asian Aquac V. 2005; May 2014:339-51.

4. Zwart MP, Dieu BTM, Hemerik L, Vlak JM. Evolutionary trajectory of white spot syndrome virus (WSSV) genome shrinkage during spread in Asia. PLoS One. 2010;5.

5. Gudkovs N, Murwantoko I, Walker PJ. Stability of the WSSV ORF94 VNTR genotype marker during passage in marine shrimp, freshwater crayfish and freshwater prawns. Dis Aquat Org. 2014;111:249-57.

6. Marks H, Goldbach RW, Vlak JM, Van Hulten MCW. Genetic variation among isolates of white spot syndrome virus. Arch Virol. 2004;149:673-97.

7. Muller IC, Andrade TPD, Tang-Nelson KFJ, Marques MRF, Lightner DV. Genotyping of white spot syndrome virus (WSSV) geographical isolates from Brazil and comparison to other isolates from the Americas. Dis Aquat Org. 2010;88:91-8.

8. Gao M, Li F, Xu L, Zhu X. White spot syndrome virus strains of different virulence induce distinct immune response in Cherax quadricarinatus. Fish Shellfish Immunol. 2014;39:17-23. https://doi.org/10.1016/j.fsi.2014.04.011.

9. Li F, Gao M, Xu L, Yang F. Comparative genomic analysis of three white spot syndrome virus isolates of different virulence. Virus Genes. 2017;53:249-58.

10. Abdollahi S, Rasooli I, Mousavi Gargari SL. An in silico structural and physicochemical characterization of TonB-dependent copper receptor in A baumannii. Microb Pathog 2018;118 2017:18-31. https://doi.org/10.1016/j. micpath.2018.03.009

11. Nagarajan V, Elasri MO. Structure and function predictions of the Msa protein in Staphylococcus aureus. BMC Bioinformatics. 2007;8(SUPPL. 7):1-9.

12. Ferron F, Bussetta C, Dutartre $H$, Canard B. The modeled structure of the RNA dependent RNA polymerase of GBV-C virus suggests a role for motif $E$ in Flaviviridae RNA polymerases. BMC Bioinformatics. 2005;6:1-16.

13. Ganguly B, Rastogi SK. Structural and functional modeling of viral protein 5 of infectious bursal disease virus. Virus Res. 2018;247 November 2017:55-60. https://doi.org/10.1016/j.virusres.2018.01.017.

14. Krupovic M, Dolja W, Koonin EV. Plant viruses of the Amalgaviridae family evolved via recombination between viruses with double-stranded and negative-strand RNA genomes. Biol Direct. 2015;10:12. https://doi.org/10. 1186/s13062-015-0047-8.

15. Ganguly B, Prasad S. Homology modeling and functional annotation of bubaline pregnancy associated glycoprotein 2. J Anim Sci Biotechnol. 2012;3:1-9.
16. Katoh K, Rozewicki J, Yamada KD. MAFFT online service: multiple sequence alignment, interactive sequence choice and visualization. Brief Bioinform. 2017; May:1-7. https://doi.org/10.1093/bib/bbx108.

17. Kearse M, Moir R, Wilson A, Stones-Havas S, Cheung M, Sturrock S, et al. Geneious basic: an integrated and extendable desktop software platform for the organization and analysis of sequence data. Bioinformatics. 2012;28:1647-9.

18. Crooks G, Hon G, Chandonia J, Brenner S. NCBI GenBank FTP Site \nWebLogo: a sequence logo generator. Genome Res. 2004;14:1188-90. https://doi.org/10.1101/gr.849004.1.

19. Altschul SF, Gish W, Miller W, Myers EW, Lipman DJ. Basic local alignment search tool. J Mol Biol. 1990;215:403-10. https://doi.org/10.1016/S00222836(05)80360-2.

20. Altschul SF, Madden TL, Schäffer AA, Zhang J, Zhang Z, Miller W, et al. Gapped BLAST and PSI-BLAST: a new generation of protein database search programs. Nucleic Acids Res. 1997:25:3389-402.

21. Eddy SR. Accelerated profile HMM searches. PLoS Comput Biol. 2011;7.

22. Remmert M, Biegert A, Hauser A, Söding J. HHblits: lightning-fast iterative protein sequence searching by HMM-HMM alignment. Nat Methods. 2012;9:173-5.

23. Söding J, Biegert A, Lupas AN. The HHpred interactive server for protein homology detection and structure prediction. Nucleic Acids Res. 2005: 33(SUPPL. 2):244-8.

24. Mirdita M, Von Den Driesch L, Galiez C, Martin MJ, Soding J, Steinegger M. Uniclust databases of clustered and deeply annotated protein sequences and alignments. Nucleic Acids Res. 2017;45:D170-6.

25. Eswar N, Webb B, Marti-Renom MA, Madhusudhan MS, Eramian D, Shen M, et al. Comparative protein structure modeling using Modeller. 2006.

26. Biasini M, Bienert S, Waterhouse A, Arnold K, Studer G, Schmidt T, et al. SWISS-MODEL: modelling protein tertiary and quaternary structure using evolutionary information. Nucleic Acids Res. 2014;42:252-8.

27. Zhang Y. I-TASSER server for protein 3D structure prediction. BMC Bioinformatics. 2008;9:1-8.

28. Kelly LA, Mezulis S, Yates C, Wass M, Sternberg M. The Phyre2 web portal for protein modelling, prediction, and analysis. Nat Protoc. 2015;10:845-58. https://doi.org/10.1038/nprot.2015-053.

29. Buchan DWA, Minneci F, Nugent TCO, Bryson K, Jones DT. Scalable web services for the PSIPRED Protein Analysis Workbench. Nucleic Acids Res. 2013:41(Web Server issue):349-57.

30. Lobley A, Sadowski MI, Jones DT. pGenTHREADER and pDomTHREADER: new methods for improved protein fold recognition and superfamily discrimination. Bioinformatics. 2009;25:1761-7.

31. Chen VB, Arendall WB, Headd JJ, Keedy DA, Immormino RM, Kapral GJ, et al. MolProbity: all-atom structure validation for macromolecular crystallography. Acta Crystallogr Sect D Biol Crystallogr. 2010;66:12-21.

32. Lovell SC, Davis IW, Adrendall WB, de Bakker PIW, Word JM, Prisant MG, et al. Structure validation by $C$ alpha geometry: phi,psi and $C$ beta deviation. Proteins-Structure Funct Genet. 2003;50 August 2002:437-50. https://doi. org/10.1002/prot.10286.

33. Pettersen EF, Goddard TD, Huang CC, Couch GS, Greenblatt DM, Meng EC, et al. UCSF chimera - a visualization system for exploratory research and analysis. J Comput Chem. 2004;25:1605-12.

34. Prilusky J, Felder CE, Zeev-Ben-Mordehai T, Rydberg EH, Man O, Beckmann JS, et al. FoldIndex@: a simple tool to predict whether a given protein sequence is intrinsically unfolded. Bioinformatics. 2005;21:3435-8.

35. Krogh A, Larsson B, Von Heijne G, Sonnhammer ELL. Predicting transmembrane protein topology with a hidden Markov model: application to complete genomes. J Mol Biol. 2001;305:567-80.

36. Petersen TN, Brunak S, Von Heijne G, Nielsen H. SignalP 4.0: discriminating signal peptides from transmembrane regions. Nat Methods. 2011;8:785-6. https://doi.org/10.1038/nmeth.1701.

37. Letunic I, Bork P. 20 years of the SMART protein domain annotation resource. Nucleic Acids Res. 2018;46:D493-6.

38. Marchler-Bauer A, Derbyshire MK, Gonzales NR, Lu S, Chitsaz F, Geer LY, et al. CDD: NCBI's conserved domain database. Nucleic Acids Res. 2015;43:D222-6.

39. de Castro E, Sigrist CJA, Gattiker A, Bulliard V, Langendijk-Genevaux PS, Gasteiger E, et al. ScanProsite: Detection of PROSITE signature matches and ProRule-associated functional and structural residues in proteins. Nucleic Acids Res. 2006;34(WEB. SERV. ISS):362-5.

40. Dinkel H, Michael S, Weatheritt RJ, Davey NE, Van Roey K, Altenberg B, et al. ELM - the database of eukaryotic linear motifs. Nucleic Acids Res. 2012;40:242-51. 
41. Kuper J, Wolski SC, Michels G, Kisker C. Functional and structural studies of the nucleotide excision repair helicase XPD suggest a polarity for DNA translocation. EMBO J. 2012;31:494-502.

42. Liu H, Rudolf J, Johnson KA, Mcmahon SA, Oke M, Mcrobbie A, et al. Europe PMC funders group structure of the DNA repair helicase XPD. Cell. 2012;133:801-12.

43. Fisher RD, Wang B, Alam SL, Higginson DS, Robinson $H$, Sundquist WI, et al. Structure and ubiquitin binding of the ubiquitin-interacting motif. J Biol Chem. 2003;278:28976-84.

44. Hofmann K, Falquet L. A ubiquitin-interacting motif conserved in components of the proteasomal and lysosomal protein degradation systems. Trends Biochem Sci. 2001;26:347-50.

45. Lin F, Huang H, Xu L, Li F, Yang F. Identification of three immediate-early genes of white spot syndrome virus. Arch Virol. 2011;156:1611-4. https://doi. org/10.1007/s00705-011-1004-1

46. Yang F, He J, Lin X, Li Q, Pan D, Xu XUN. Complete genome sequence of the shrimp white spot bacilliform virus complete genome sequence of the shrimp white spot bacilliform virus. J Virol. 2001:75:11811-20.

47. Aspenström P. Formin-binding proteins: modulators of formin-dependent actin polymerization. Biochim Biophys Acta Mol Cell Res. 2010;1803:174-82. https://doi.org/10.1016/j.bbamcr.2009.06.002.

48. Otomo T, Tomchick DR, Otomo C, Panchal SC, Machius M, Rosen MK Structural basis of actin filament nucleation and processive capping by a formin homology 2 domain. Nature. 2005;433:488-94.

49. Otomo T, Tomchick DR, Otomo C, Machius M, Rosen MK. Crystal structure of the formin mDIA1 in autoinhibited conformation. PLoS One. 2010;5:1-13.

50. Xu Y, Moseley JB, Sagot I, Poy F, Pellman D, Goode BL, et al. Crystal structures of a formin homology-2 domain reveal a tethered dimer architecture. Cell. 2004;116:711-23.

51. Schönichen A, Geyer M. Fifteen formins for an actin filament: a molecular view on the regulation of human formins. Biochim Biophys Acta Mol Cell Res. 2010;1803:152-63. https://doi.org/10.1016/j.bbamcr.2010.01.014.

52. Thompson ME, Heimsath EG, Gauvin TJ, Higgs HN, Jon KF. FMNL3 FH2-actin structure gives insight into formin-mediated actin nucleation and elongation. Nat Struct Mol Biol. 2013;20:111-8.

53. Spear M, Wu Y. Viral exploitation of actin: force-generation and scaffolding functions in viral infection. Virol Sin. 2014;29:139-47.

54. Aggarwal A, lemma TL, Shih I, Newsome TP, McAllery S, Cunningham AL, et al. Mobilization of HIV spread by diaphanous 2 dependent filopodia in infected dendritic cells. PLoS Pathog. 2012;8.

55. Alvarez DE, Agaisse $H$. The formin FHOD1 and the small GTPase Rac1 promote vaccinia virus actin-based motility. J Cell Biol. 2013;202:1075-90.

56. Han F, Xu J, Zhang X. Characterization of an early gene (wsv477) from shrimp white spot syndrome virus (WSSV). Virus Genes. 2007;34:193-8.

57. Huang $T$, Xu D, Zhang X. Characterization of host microRNAs that respond to DNA virus infection in a crustacean. BMC Genomics. 2012;13.

58. Huang $T$, Zhang $X$. Functional analysis of a crustacean MicroRNA in host-virus interactions. J Virol. 2012;86:12997-3004. https://doi.org/10.1128/JVI.01702-12.

59. Conte MR. Structure of tandem RNA recognition motifs from polypyrimidine tract binding protein reveals novel features of the RRM fold. EMBO J. 2000; 19:3132-41. https://doi.org/10.1093/emboj/19.12.3132.

60. Kondo Y, Oubridge C, Van Roon AM, Nagai K. Crystal structure of human U1 snRNP, a small nuclear ribonucleoprotein particle, reveals the mechanism of 5 ' splice site recognition. Elife. 2015:4:1-19. https://doi.org/10.7554/eLife.04986.

61. Maris C, Dominguez C, Allain FHT. The RNA recognition motif, a plastic RNAbinding platform to regulate post-transcriptional gene expression. FEBS J. 2005; 272:2118-31.

62. Martin-Tumasz S, Richie AC, Clos L, Brow DA, Butcher SE. A novel occluded RNA recognition motif in Prp24 unwinds the U6 RNA internal stem loop. Nucleic Acids Res. 2011;39:7837-47.

63. Weber G, Trowitzsch S, Kastner B, Lührmann R, Wahl MC. Functional organization of the Sm core in the crystal structure of human U1 SnRNP. EMBO J. 2010;29:4172-84.

64. Kim CS, Seol SK, Song O-K, Park JH, Jang SK. An RNA-binding protein, hnRNP A1, and a scaffold protein, Septin 6, facilitate hepatitis C virus replication. J Virol. 2007:81:3852-65. https://doi.org/10.1128/JVI.01311-06.

65. Cléry A, Blatter M, Allain FHT. RNA recognition motifs: boring? Not quite Curr Opin Struct Biol. 2008;18:290-8.

66. Liu Y, Wu J, Song J, Sivaraman J, Hew CL. Identification of a novel nonstructural protein, VP9, from white spot syndrome virus: its structure reveals a ferredoxin fold with specific metal binding sites. J Virol. 2006:80: 10419-27. https://doi.org/10.1128/JVI.00698-06.
67. Chen J, Skehel JJ, Wiley DC. N- and C-terminal residues combine in the fusion-pH influenza hemagglutinin HA2 subunit to form an $\mathrm{N}$ cap that terminates the triple-stranded coiled coil. Proc Natl Acad Sci. 1999;96:896772. https://doi.org/10.1073/pnas.96.16.8967.

68. Wang W, DeFeo CJ, Alvarado-Facundo E, Vassell R, Weiss CD. Intermonomer interactions in hemagglutinin subunits HA1 and HA2 affecting hemagglutinin stability and influenza virus infectivity. J Virol. 2015;89:1060211. https://doi.org/10.1128/JVI.00939-15.

69. Ramos-Paredes J, Grijalva-Chon JM, la Rosa-Vélez JD, Enríquez-Paredes LM. New genetic recombination in hypervariable regions of the white spot syndrome virus isolated from Litopenaeus vannamei (Boone) in Northwest Mexico. Aquac Res. 2012;43:339-48. https://doi.org/10.1111/j. 1365-2109.2011.02836.x.

70. Shekar M, Pradeep B, Karunasagar I. White spot syndrome virus: genotypes, epidemiology and evolutionary studies. Indian J Virol. 2012;23:175-83.

71. Huang C. Proteomic analysis of shrimp white spot syndrome viral proteins and characterization of a novel envelope protein VP466. Mol Cell Proteomics. 2002;1:223-31. https://doi.org/10.1074/mcp.M100035-MCP200.

72. Lange A, Mills RE, Lange CJ, Stewart M, Devine SE, Corbett AH. Classical nuclear localization signals: definition, function, and interaction with importin ?? J Biol Chem. 2007;282:5101-5.

73. Cokol M, Nair R, Rost B. Finding nuclear localization signals. EMBO Rep. 2000;1:411-5. https://doi.org/10.1093/embo-reports/kvd092.

74. Marfori M, Mynott A, Ellis JJ, Mehdi AM, Saunders NFW, Curmi PM, et al. Molecular basis for specificity of nuclear import and prediction of nuclear localization. Biochim Biophys Acta Mol Cell Res. 1813;2011:1562-77. https:// doi.org/10.1016/j.bbamcr.2010.10.013.

75. Riddick G, Macara IG. The adapter importin-a provides flexible control of nuclear import at the expense of efficiency. Mol Syst Biol. 2007;3:1-7. https://doi.org/10.1038/msb4100160.

76. Sage V Le, Mouland AJ. Viral Subversion of the Nuclear Pore Complex. Viruses. 2013;5(8):2019-42. Published online 2013 Aug 15. doi: https:// doi.org/10.3390/v5082019.

77. Dieu BTM. Molecular epidemiology of white spot syndrome virus within Vietnam. J Gen Virol. 2004;85:3607-18. https://doi.org/10.1099/vir.0.80344-0.

78. Parra RG, Espada R, Verstraete N, Ferreiro DU. Structural and energetic characterization of the Ankyrin repeat protein family. PLoS Comput Biol. 2015;11:1-20.

79. Li J, Mahajan A, Tsai MD. Ankyrin repeat: a unique motif mediating proteinprotein interactions. Biochemistry. 2006;45:15168-78.

80. Sedgwick SG, Smerdon SJ. The ankyrin repeat: a diversity of interactions on a common structural framework. Trends Biochem Sci. 1999:24:311-6.

81. Herbert MH, Squire CJ, Mercer AA. Poxviral ankyrin proteins. Viruses. 2015;7:709-38,

82. Noel EA, Kang M, Adamec J, Van Etten JL, Oyler GA. Chlorovirus Skp1binding Ankyrin repeat protein interplay and mimicry of cellular ubiquitin ligase machinery. J Virol. 2014;88:13798-810. https://doi.org/10.1128/JVl. 02109-14.

83. Wang Z, Chua HK, Gusti AARA, Fenner B, Manopo I, Wang H, et al. RING-H2 Protein WSSV249 from White Spot Syndrome Virus Sequesters a Shrimp Ubiquitin-Conjugating Enzyme, PvUbc , for Viral Pathogenesis RING-H2 Protein WSSV249 from White Spot Syndrome Virus Sequesters a Shrimp Ubiquitin-Conjugating Enzyme, PvUbc, for . 2005;79:8764-8772.

84. Zheng N, Shabek N. Ubiquitin Ligases: Structure, Function, and Regulation. Annu Rev Biochem. 2017;86:129-157. doi: https://doi.org/10.1146/annurevbiochem-060815-014922. Epub 2017 Mar 27.

\section{Ready to submit your research? Choose BMC and benefit from:}

- fast, convenient online submission

- thorough peer review by experienced researchers in your field

- rapid publication on acceptance

- support for research data, including large and complex data types

- gold Open Access which fosters wider collaboration and increased citations

- maximum visibility for your research: over $100 \mathrm{M}$ website views per year

At $\mathrm{BMC}$, research is always in progress.

Learn more biomedcentral.com/submission 